\title{
Utility of Endobronchial Ultrasound-Guided Transbronchial Needle Aspiration in Diagnosis of Intrathoracic Lymphadenopathy in Patients with Human Immunodeficiency Virus Infection
}

\author{
Audrey Yan Yi Han, Aik Hau Tan, and Mariko Siyue Koh \\ Department of Respiratory and Critical Care Medicine, Singapore General Hospital, 20 College Road, Academia, Singapore 169856 \\ Correspondence should be addressed to Aik Hau Tan; tan.aik.hau@sgh.com.sg
}

Received 25 June 2014; Revised 18 September 2014; Accepted 27 September 2014

Academic Editor: Andrea Zanini

Copyright (C) 2015 Audrey Yan Yi Han et al. This is an open access article distributed under the Creative Commons Attribution License, which permits unrestricted use, distribution, and reproduction in any medium, provided the original work is properly cited.

\begin{abstract}
Objective. Intrathoracic lymphadenopathy (LAD) in patients with Human Immunodeficiency Virus (HIV) infection is common, with wide-ranging diagnoses, from benign to malignant causes. Endobronchial Ultrasound-guided Transbronchial Needle Aspiration (EBUS-TBNA) is a relatively new technology with established applications in lung cancer, sarcoidosis, and tuberculosis. We sought to find out whether the addition of EBUS-TBNA to the diagnostic algorithm for LAD in HIV patients will reduce the need for mediastinoscopy. Methods. Retrospective chart review of all EBUS-TBNA procedures performed in our centre from August 2008 to December 2012. Results. 513 patients had EBUS-TBNA performed during this period. We identified nine HIV-infected patients who had LAD of unknown cause and underwent EBUS-TBNA. The procedure reduced the need for mediastinoscopy in eight patients (89\%). Conclusions. Potential mediastinoscopies can be avoided by utilising EBUS-TBNA in HIV patients with LAD.
\end{abstract}

\section{Introduction}

Intrathoracic lymphadenopathy (LAD) is a challenging problem in patients infected with Human Immunodeficiency Virus (HIV). Jasmer et al. demonstrated an incidence of $35 \%$ based on routine computed tomography (CT) scan, performed for a cohort of 318 patients with HIV infection [1]. Diagnoses are extensive, ranging from commoner diagnoses of Mycobacterial infections (tuberculous and nontuberculous) and malignancies (lymphoma, primary lung cancer, Kaposi's sarcoma) to less common diagnoses of reactive lymphadenopathy in infection (bacterial, fungal, Pneumocystis jirovecii pneumonia previously known as Pneumocystis carinii pneumonia) and nonspecific lymphoid hyperplasia $[1,2]$.

Conventionally, patients would undergo mediastinoscopy or open biopsy via thoracotomy, which incur higher morbidity and a risk of general anaesthesia as compared to bronchoscopic procedures [3]. Conventional Transbronchial Needle Aspiration (TBNA) via bronchoscopy was an alternative method of diagnosis, but with a diagnostic rate of only 52\% when performed in this group of patients [4]. Endobronchial Ultrasound-guided Transbronchial Needle Aspiration (EBUS-TBNA) is a relatively new technology which uses real-time linear ultrasound imaging to guide intrathoracic lymph node and lung mass tissue sampling during bronchoscopy. Its utility in the diagnosis of lung cancer [5], sarcoidosis [6], and tuberculosis [7] has been proven; in particular, it has been shown to have significantly better sensitivity and yield than conventional TBNA for lung cancer and sarcoidosis $[5,6]$. Our aim was to find out if addition of EBUS-TBNA into the diagnostic algorithm for LAD in HIV patients will reduce the need for mediastinoscopy.

\section{Methods}

Retrospective chart review of all EBUS-TBNA procedures performed in our centre from August 2008 to December 2012 was carried out. Completeness of data was ensured with the 


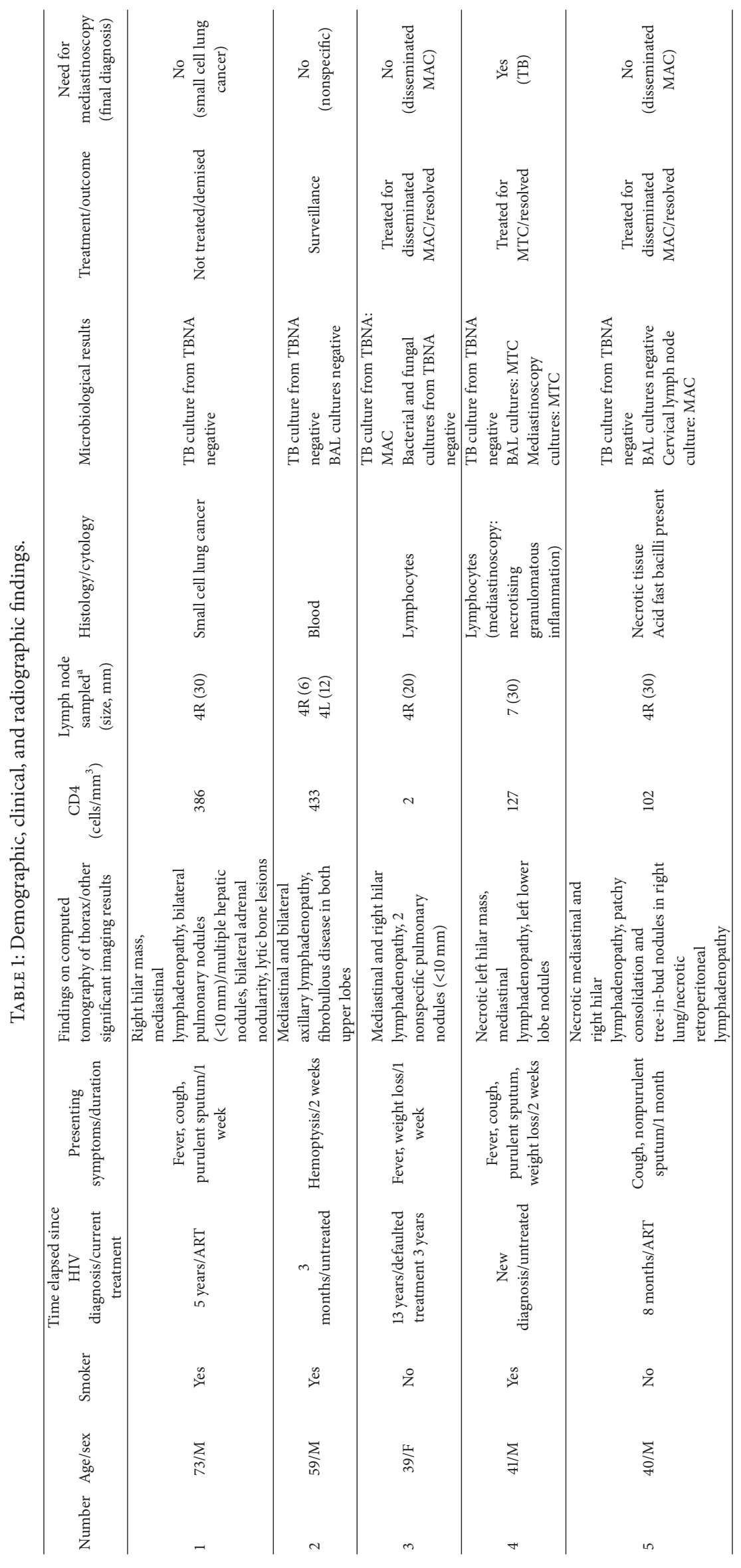




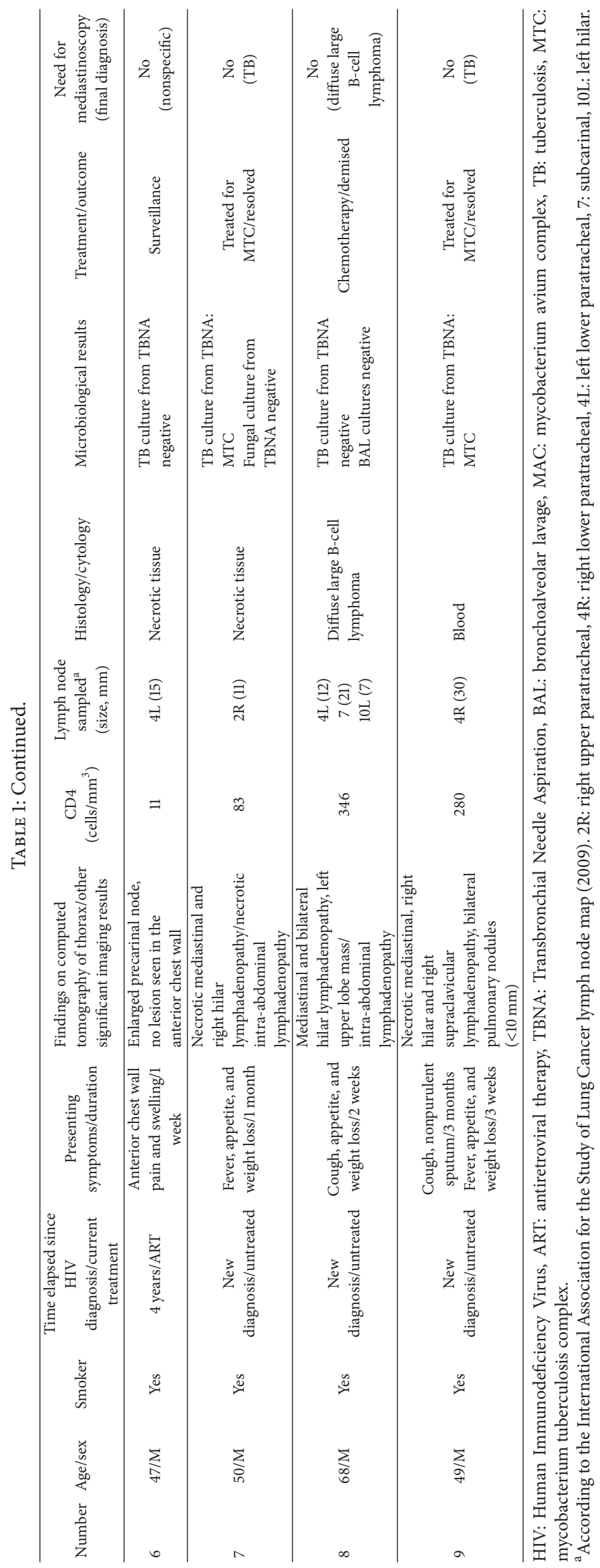


use of a centralized database that was set up at the time of introduction of EBUS-TBNA to our institution. The study was approved by our Centralized Institutional Review Board (2008/458/B). All cases of HIV infection were confirmed by Western blot. Demographic data, clinical presentations, results, clinical management, and outcomes are summarised in Table 1.

Informed consent was obtained from each patient prior to the procedure. EBUS-TBNA was carried out in our endoscopy suite under local anaesthesia and moderate sedation with midazolam and/or fentanyl.

\section{Results}

513 patients had EBUS-TBNA performed during this period. We identified nine HIV-infected patients who had LAD of unknown cause that underwent EBUS-TBNA. The average age of these HIV-infected patients was 52 years (range: 40 to 73 years) and the average CD4 count was 197 (range: 2 to 433 cells $/ \mathrm{mm}^{3}$ ). The most common presenting symptom was fever and the paratracheal lymph nodes were the most frequently sampled. The addition of EBUS-TBNA reduced the need for mediastinoscopy in eight out of nine patients (Table 1).

All 5 cases of Mycobacterial infection were treated with Mycobacterial therapy, with clinical and radiological improvement. One patient with small cell lung cancer died before treatment was commenced (Patient 1). One patient with diffuse large B-cell lymphoma received chemotherapy but died 10 weeks later from progressive disease (Patient 8 ) (Table 1).

Although an indeterminate result was recorded for Patient 2, he remains clinically well 22 months after EBUSTBNA. Follow-up CT thorax 22 months later revealed stable mediastinal lymphadenopathy, partial resolution of axillary lymphadenopathy, and no new findings. Thus, he was deemed to have nonspecific mediastinal lymphadenopathy. Similarly, Patient 6 remains clinically well on follow-up 24 months after EBUS-TBNA.

One complication was encountered. Patient 1 had an uneventful bronchoscopic procedure, but desaturated and had an asystolic collapse 18 hours thereafter. Chest radiograph did not reveal any pneumothorax or new consolidation. He did not have any haemoptysis to suggest bleeding as a complication of the procedure. The cause of death was attributed to an acute myocardial event, as he had significant cardiovascular risk factors (diabetes, hypertension, smoker). The case was referred to the coroner's office but an autopsy was not performed.

\section{Discussion}

We found that EBUS-TBNA decreased the need for mediastinoscopy in eight out of nine (89\%) HIV patients with LAD. EBUS-TBNA was able to diagnose four out of five cases of lymphadenopathy secondary to Mycobacterial infections, one case of diffuse large B-cell lymphoma and one case of small cell lung cancer. It was nondiagnostic in one case of tuberculosis (diagnosed by mediastinoscopy). Although a definitive diagnosis was not obtained in two patients, these patients had relatively small lymph node enlargement $(\leq 15 \mathrm{~mm})$, which likely represented an inflammatory reaction to HIV infection, rather than actual lymph node involvement (i.e., "true negative" EBUS-TBNA procedures). Stability in their conditions after 22 months and 24 months of followup further supports the conclusion that the lymphadenopathy observed was unlikely to be clinically significant.

To our knowledge, this is the first series describing the utility of EBUS-TBNA in the diagnosis of LAD in HIVinfected patients, and our findings support the utilisation of this bronchoscopic technique in this group of patients.

Although there was a patient who died 18 hours after bronchoscopy, this was likely due to the sedation and/or hypoxemia related to bronchoscopy, rather than EBUSTBNA itself. Major complications from bronchoscopy are uncommon $(0.08 \%$ to $0.5 \%)$, and so is mortality (up to $0.04 \%$ ) [8]. Based on a nation-wide survey involving 520 centres in Japan, complications arising from EBUS-TBNA are also uncommon $(1.2 \%)$, and mortality is rare $(0.01 \%)$ [9]. The main complications were bleeding $(0.68 \%)$ and infection $(0.19 \%)$ [9]. Our patient's significant cardiovascular risk factors of diabetes, hypertension, and smoking, as well as the events leading to his demise, strongly suggest an acute myocardial event. In general, complication and mortality rates from bronchoscopy $(0.08-0.5 \%$ and $0.04 \%$, resp.) and EBUS-TBNA ( $1.2 \%$ and $0.01 \%$, resp.) are still comparable to or lower than those of mediastinoscopy $(1.07 \%$ and $0.05 \%$, resp.) [3]. In addition, EBUS-TBNA does not require general anaesthesia or an inpatient hospitalization in most centres.

The main limitations of our study are its retrospective nature and small numbers. However, completeness of data is ensured by the centralized database that was set up at the time of introduction of EBUS-TBNA to our institution, as well as cross-checking of results with the Electronic Health Records. Secondly, patients referred for EBUS-TBNA were screened for HIV infection only when there was clinical suspicion of the disease. We recognise that there may be patients who have undergone EBUS-TBNA with concomitant undiagnosed HIV infection. Nevertheless, as a tertiary referral centre, we have a low threshold for ordering HIV testing in patients. In fact, 33.3\% (171 of 513 patients) of our EBUS-TBNA cohort had undergone HIV testing. Therefore, we believe that we are unlikely to have underdiagnosed HIV infection in our cohort of patients, and that our findings are representative of real-life clinical practice.

In conclusion, it appears that potential mediastinoscopies can be avoided by utilising EBUS-TBNA in HIV patients with intrathoracic lymphadenopathy.

\section{Conflict of Interests}

The authors declare that there is no conflict of interests regarding the publication of this paper. 


\section{References}

[1] R. M. Jasmer, M. B. Gotway, J. M. Creasman, W. R. Webb, K. J. Edinburgh, and L. Huang, "Clinical and radiographic predictors of the etiology of computed tomography-diagnosed intrathoracic lymphadenopathy in HIV-infected patients," Journal of Acquired Immune Deficiency Syndromes, vol. 31, no. 3, pp. 291298, 2002.

[2] P. Wannakrairot, T. Y.-M. Leong, and A. S.-Y. Leong, "The morphological spectrum of lymphadenopathy in HIV infected patients," Pathology, vol. 39, no. 2, pp. 223-227, 2007.

[3] A. Lemaire, I. Nikolic, T. Petersen et al., "Nine-year single center experience with cervical mediastinoscopy: complications and false negative rate," Annals of Thoracic Surgery, vol. 82, no. 4, pp. 1185-1190, 2006.

[4] T. J. Harkin, C. Ciotoli, D. J. Addrizzo-Harris, D. P. Naidich, J. Jagirdar, and W. N. Rom, "Transbronchial needle aspiration (TBNA) in patients infected with HIV," American Journal of Respiratory and Critical Care Medicine, vol. 157, no. 6, part 1, pp. 1913-1918, 1998.

[5] M. B. Wallace, J. M. S. Pascual, M. Raimondo et al., "Minimally invasive endoscopic staging of suspected lung cancer," Journal of the American Medical Association, vol. 299, no. 5, pp. 540-546, 2008.

[6] A. Tremblay, D. R. Stather, P. MacEachern, M. Khalil, and S. K. Field, "A randomized controlled trial of standard vs endobronchial ultrasonography-guided transbronchial needle aspiration in patients with suspected sarcoidosis," Chest, vol. 136, no. 2, pp. 340-346, 2009.

[7] N. Navani, P. L. Molyneaux, R. A. Breen et al., "Utility of endobronchial ultrasound-guided transbronchial needle aspiration in patients with tuberculous intrathoracic lymphadenopathy: a multicentre study," Thorax, vol. 66, no. 10, pp. 889-893, 2011.

[8] British Thoracic Society Bronchoscopy Guidelines Committee, "British thoracic society guidelines on diagnostic flexible bronchoscopy," Thorax, vol. 56, supplement 1, pp. i1-i21, 2001.

[9] F. Asano, M. Aoe, Y. Ohsaki et al., "Complications associated with endobronchial ultrasound-guided transbronchial needle aspiration: a nationwide survey by the Japan Society for Respiratory Endoscopy," Respiratory Research, vol. 14, no. 1, article 50, 2013. 


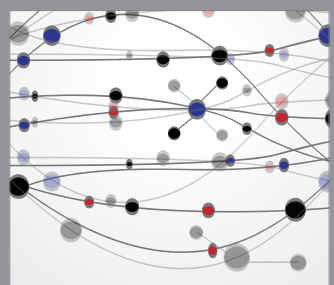

The Scientific World Journal
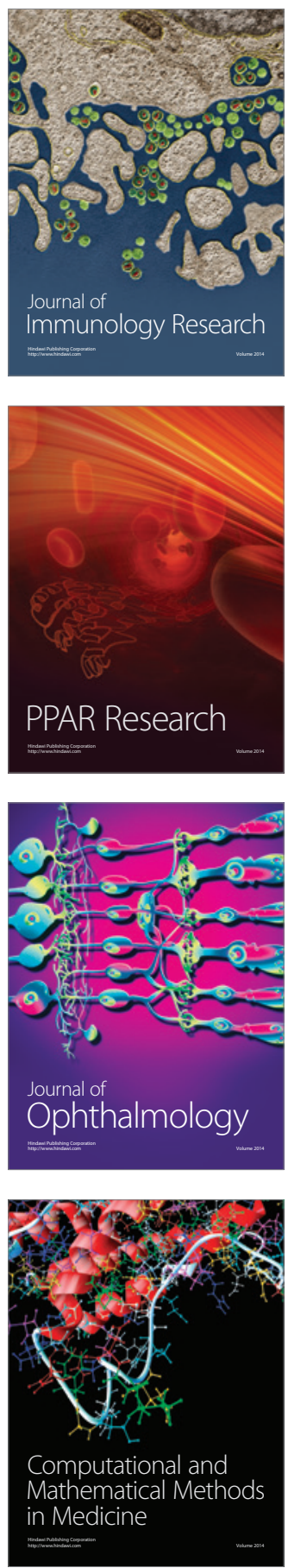

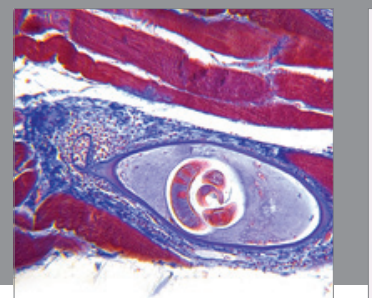

Gastroenterology

Research and Practice
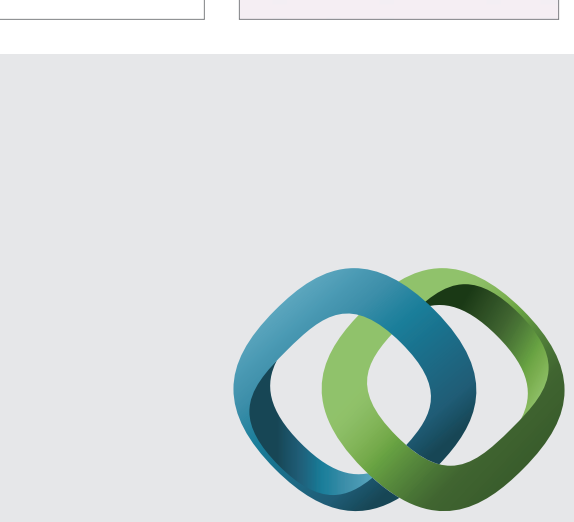

\section{Hindawi}

Submit your manuscripts at

http://www.hindawi.com
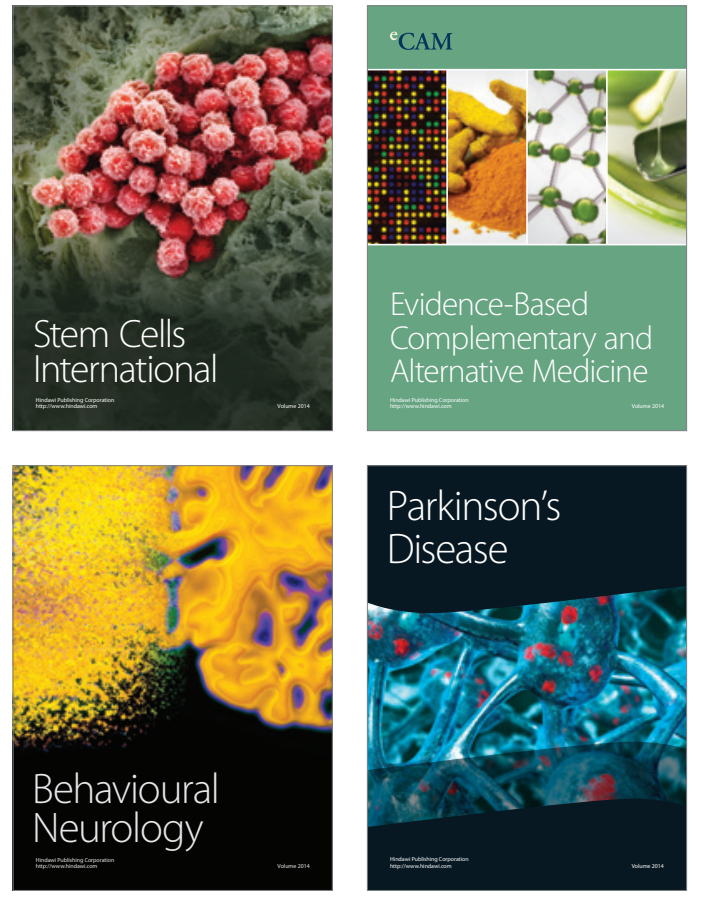
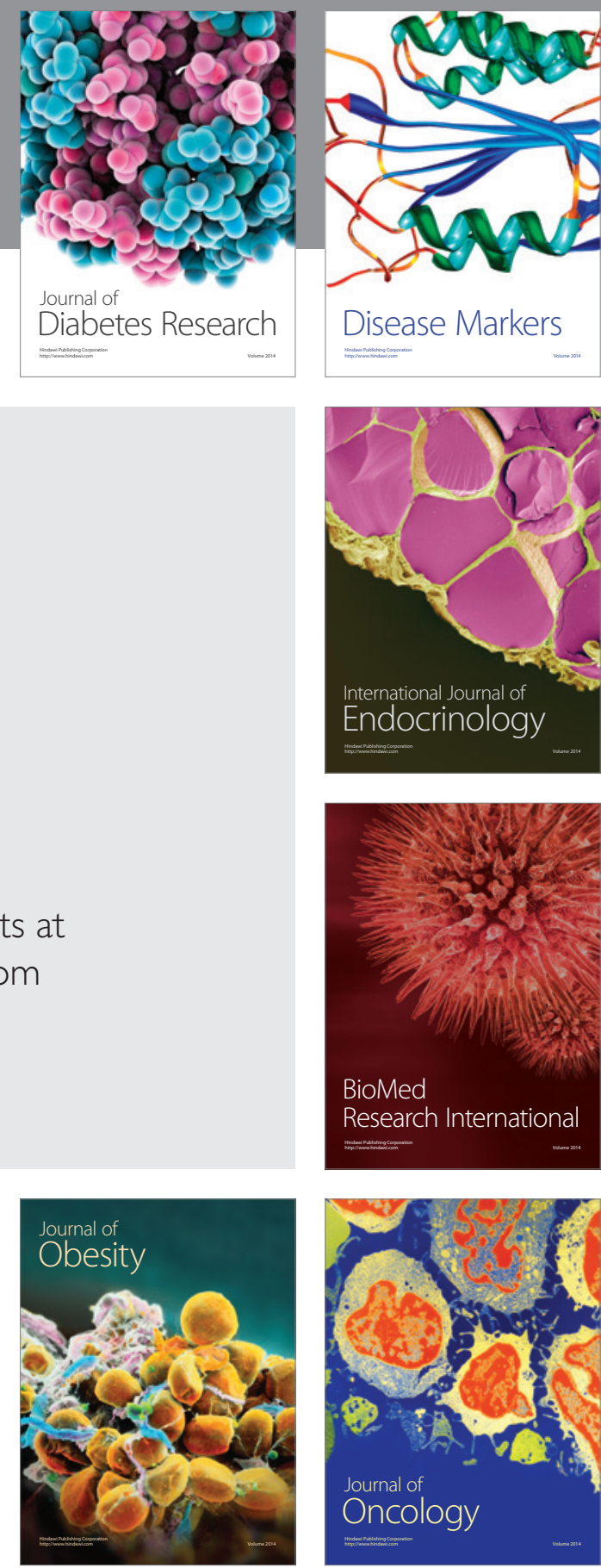

Disease Markers
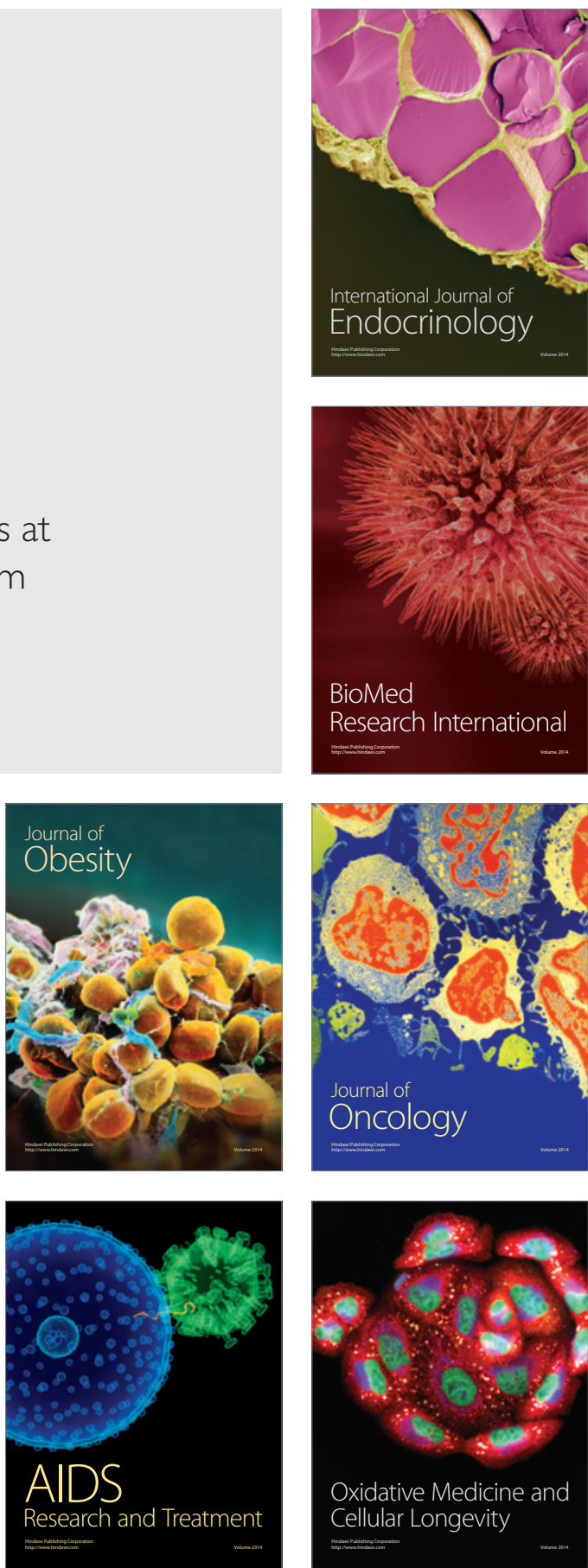